\title{
Influence of cognitive reserve in schizophrenia: A systematic review
}

\author{
Paula Herrero $^{\mathrm{a}}$, Israel Contador ${ }^{\mathrm{b}, *}$, Yaakov Stern ${ }^{\mathrm{c}}$, Bernardino Fernández-Calvo ${ }^{\mathrm{d}}$, \\ Abraham Sánchez ${ }^{\mathrm{b}}$, Francisco Ramos ${ }^{\mathrm{e}}$ \\ ${ }^{\text {a }}$ Psychiatric Institute José Germain, Madrid, Spain \\ ${ }^{\mathrm{b}}$ Department of Basic Psychology, Psychobiology and Methodology of Behavioral Sciences, Faculty of Psychology, University of Salamanca, Spain \\ ${ }^{\mathrm{c}}$ Departments of Neurology, Psychiatry, and Psychology, and the Taub Institute for the Research on Alzheimer's Disease and the Aging Brain, Columbia University College of \\ Physicians and Surgeons, New York, United States \\ ${ }^{\mathrm{d}}$ Department of Psychology, Federal University of Paraíba, Brazil \\ ${ }^{\mathrm{e}}$ Department of Personality, Assessment and Psychological Treatment, Faculty of Psychology, University of Salamanca, Spain
}

\section{A R T I C L E I N F O}

\section{Keywords:}

Schizophrenia

Cognitive reserve

Cognitive rehabilitation

\begin{abstract}
A B S T R A C T
Objective: To perform a systematic review of the influence of sociobehavioural indicators of cognitive reserve (CR) in the risk of schizophrenia, the clinical manifestations of the disease, and cognitive intervention programs (CRT) carried out with these patients.

Method: A cross search was made by two independent reviewers in Pubmed and PsycINFO databases using keywords "schizophrenia" and "cognitive reserve." Twenty-one studies which analyzed different CR proxies were selected and the level of evidence was classified according to the Oxford Centre for Evidence-Based Medicine.

Result: People with higher CR may have a lower risk of developing schizophrenia and a later onset of disease. In addition, they present better neuropsychological and functional performance in the illness course. However, the suspected influence of CR on the effectiveness of CRT in patients with schizophrenia is currently unresolved.

Conclusion: Our findings suggest that higher CR delays the clinical diagnosis threshold and severity of the symptoms in patients with schizophrenia. However, effect of singular sociobehavioral measures on clinical expression and benefits of intervention program need further investigation.
\end{abstract}

\section{Introduction}

The lack of a direct link between the degree of brain pathology and clinical manifestations has given rise to the term "reserve" as a moderator of this relationship (Stern, 2012). In this sense, there are two types of models. From passive models, Katzman (1993) proposed the term brain reserve. This concept refers to individual differences in the brain structure, such as its size and other quantitative characteristics (number of neurons, synapses, or dendritic branches), which would allow the individual to better cope with neuropathology. On the other hand, the active models refer to cognitive reserve (CR), defined by Stern (2002) as the ability to optimize cognitive performance through the use of alternative neural networks, or through the more efficient use of already existing networks, which could reflect the use of different cognitive strategies when the habitual ones do not produce the desired result. Essentially, CR is theoretical construct which is estimated based on socio-behavioral proxies (e.g., premorbid IQ or education), residual approaches (i.e., cognitive not explained by sociodemographic or brain predictors) and/or neuroimaging techniques (see Stern et al., 2018). Possibly, functional activation brain networks are the closest direct measure of $\mathrm{CR}$, but even studies which using this approach cannot assure a direct measure of CR.

The development of both types of reserve is closely related with brain plasticity, a term that reflects the brain's ability to adapt functionally and structurally in response to a constantly changing environment (Burke and Barnes, 2006). Starting from this concept, the active models propose that $\mathrm{CR}$ is based on the application of resources acquired due to educational experience, profession or premorbid intelligence (Stern et al., 1999). Thus, different experiences throughout life can separately contribute to CR, which may vary based on the individual's experiences (Jiménez, 2016). Life experiences that may participate in the accumulation of CR have been studied mostly in Alzheimer's disease (AD) and other dementias. Thus, sufficient evidence has been compiled to state that years of education, occupational attainment, social and leisure activities, mental/cognitive activities, IQ, and even physical activity act as protective factors against the

\footnotetext{
* Corresponding author at: Facultad de Psicología, Universidad de Salamanca, Avda, de la Merced 109-131, ES-37005, Salamanca, Spain.

E-mail address: icontador@usal.es (I. Contador).
} 
development of dementia (Contador et al., 2015; Llamas-Velasco et al., 2015; Scarmeas et al., 2001; Valenzuela and Sachdev, 2005). However, and contrary to what one might expect, it has been reported that when $\mathrm{AD}$ emerges, people who have a high $\mathrm{CR}$ suffer a more severe decline after the diagnosis and die sooner, due to their greater degree of brain neuropathology when the disease is detected (Contador et al., 2017; Stern et al., 1999). Finally, it has been shown that cognitive interventions can delay the accelerated cognitive decline in people with mild $\mathrm{AD}$ and high $\mathrm{CR}$ (educational level) over short intervals (Contador et al., 2016), which partially supports the hypothesis of greater plasticity and compensation in people with high CR.

In this context, Barnett et al. (2006) have advocated that the CR may also influence mental disorders such as schizophrenia, acting on the risk of the development and expression of symptoms. However, there are few studies in this area and the applicability of the CR in mental disorders is controversial.

Schizophrenia is a complex and severe mental illness that manifests through neurocognitive dysfunction (Rund, 2009) and is characterized by a heterogeneous course (Lewis and Lieberman, 2000). Its etiology is unknown, although there are various explanatory hypotheses that are not necessarily in opposition (Mané et al., 2009). The neurodevelopmental hypothesis posits a change in the brain's development, altered by genetic or environmental factors in patients with schizophrenia (Rund, 2009). This idea is supported by studies indicating the presence of neurological, cognitive, behavioral, and functional difficulties in early stages of schizophrenia - even before the onset of clinical symptoms (Davidson et al., 1999; Green et al., 1989; Jones et al., 1998, 1994; Kenny et al., 1997; Sørensen et al., 2003), as well as reduced volume of the brain (Vita et al., 2006) at the onset of the disease. In addition, it has been shown that some of the grey-matter abnormalities associated with psychotic disorders predate the onset of frank symptoms (Pantelis et al., 2003). These studies indicate that brain dysfunction occurs before the onset of the disease, and that the severity of brain dysfunction helps determine the age at which the cognitive deficits become apparent (Bora, 2015). This evidence is consistent with DeLisi (1992), who claimed that younger age at onset of psychosis is an indicator of a more severe level of pathology and is linked to poor prognosis.

The neurodegenerative hypothesis, on the other hand, suggests that the pathological changes in brain morphology occur along with or after disease onset (early stages) and may be associated with the course and the severity of the disease (Lieberman et al., 1997). Supporting this idea, progressive decreases in gray matter in the frontal and temporal areas have been found during the first four years after the onset of schizophrenia (Mané et al., 2009). In particular, Jacobsen et al. (1998) documented a $7 \%$ annual reduction of hippocampal volume in these patients. Additionally, Ho et al. (2003) observed that frontal white matter decreases after the onset of the disease, whereas Rapoport et al. (1997) found that ventricular volume increased by $10 \%$ each year. However, these findings of brain degeneration do not coincide with many reports indicating that a significant percentage of these patients (23-73\%) do not present neuropsychological alterations (Bryson et al., 1993; Kremen et al., 2000). Moreover, longitudinal studies report that there is no worsening in neurocognitive functions after the onset of the disease (Hoff et al., 2005; Kurtz, 2005; Rund, 2009, 1998), some of them have even found a clinical improvement while brain structures (larger ventricles, smaller hemispheres) were affected (DeLisi et al., 1998; Gur et al., 1998; Jacobsen et al., 1998). In the same vein, Sporn et al. (2003) found that greater worsening of positive and negative symptoms was significantly related to a higher rate of gray matter reduction. Finally, a later study followed patients with schizophrenia for 2 years, finding a progressive cortical narrowing of the frontal cortex and, to a lesser extent, in the superior temporal cortex, in the absence of clinical or cognitive impairment during the time (Gutiérrez-Galve et al., 2015).

These results highlight a lack of correspondence between brain pathology and clinical symptoms associated with schizophrenia, suggesting the presence of other factors that can influence this association. In this sense, CR is a factor that could buffer the effect of neuropathology, explaining the heterogeneous course observed in these patients. Related to this issue, lower CR has been associated with the presence of negative symptoms (Cohen et al., 2007; Harvey et al., 2006; Konstantakopoulos et al., 2011) and the lack of cognitive improvement at follow-up (Bora and Murray, 2014). In the same line, better cognitive performance at baseline is related to better effects in practice (Rapport et al., 1997), which could lead us to think that patients with lower CR would show less improvement than those with higher CR. Learning more about the role of $\mathrm{CR}$ in patients with schizophrenia could contribute to prevention strategies for the onset of this disorder and potentially improve the existing intervention strategies. However, few studies have examined the impact of CR in schizophrenia.

This work aims to undertake a systematic review of the reports studying the influence of $\mathrm{CR}$, measured through multiple indicators, on the early onset and manifestations (cognitive, functional, and psychopathological) of schizophrenia. We hypothesized that higher CR: (1) acts as a protective factor against the development of schizophrenia and (2) reduces the impact of the clinical manifestations of the disease. We also propose (3) that patients with schizophrenia with high CR will show more improvement after receiving cognitive intervention programs versus those with lower $\mathrm{CR}$.

\section{Methods}

The appraisal for this systematic review was based on AMSTAR-2 criteria for healthcare interventions (Shea et al., 2017). We used two keywords namely "schizophrenia" and "cognitive reserve" that were cross-searched by two independent reviewers on Pubmed and PsycINFO databases. Two independent raters selected eligible papers, achieving a percentage of initial agreement over 95 percent. The associated bibliography of target papers was also considered for potential inclusion and any discrepancy between reviewers was resolved by a third referee.

For this review, we selected papers in which the relationship between CR and schizophrenia was investigated in terms of the influence of sociobehavioral indicators on early diagnosis, clinical symptoms or intervention. Thus, all observational and interventions studies describing group comparisons (e.g., high vs. low reserve; interventions vs. control) and corresponding effect of CR proxies on the outcome (cognitive, behavioral or functional) were included. However, studies based exclusively on neuroimaging, those out of main topic and those related to $\mathrm{CR}$ in people with other mental or neurological disorders other than schizophrenia were excluded. We also eliminated papers not written in English. Considering these criteria, 21 empirical studies were selected for the review. A flowchart of the selection process is presented (see Fig. 1) following PRISMA guidelines (The PRISMA Group, 2009).

Finally, the quality of the evidence was assessed using the Oxford Centre for Evidence-Based Medicine (OCEBM Levels of Evidence Working Group, 2011). Five levels were considered: 1) Systematic review of randomized trials or n-of-1 trials; 2) Randomized trial or observational study with dramatic effect; 3) Non-randomized controlled cohort/follow-up study; 4) Case-series, case-control studies, or historically controlled studies; 5) Mechanism-based reasoning. Considering that well-designed observational studies may have value similar to that of experimental studies (Concato et al., 2000), level of evidence was raised from 2 to 3 level for well-designed prospective population-based studies after careful consideration. Two independent raters assessed each study, and three discrepancies were found. Then, a discussion took place to resolve divergences, consulting a third rater if necessary.

\section{Results}

Table 1 summarizes the main characteristics of the selected studies and the quality of the evidence. 


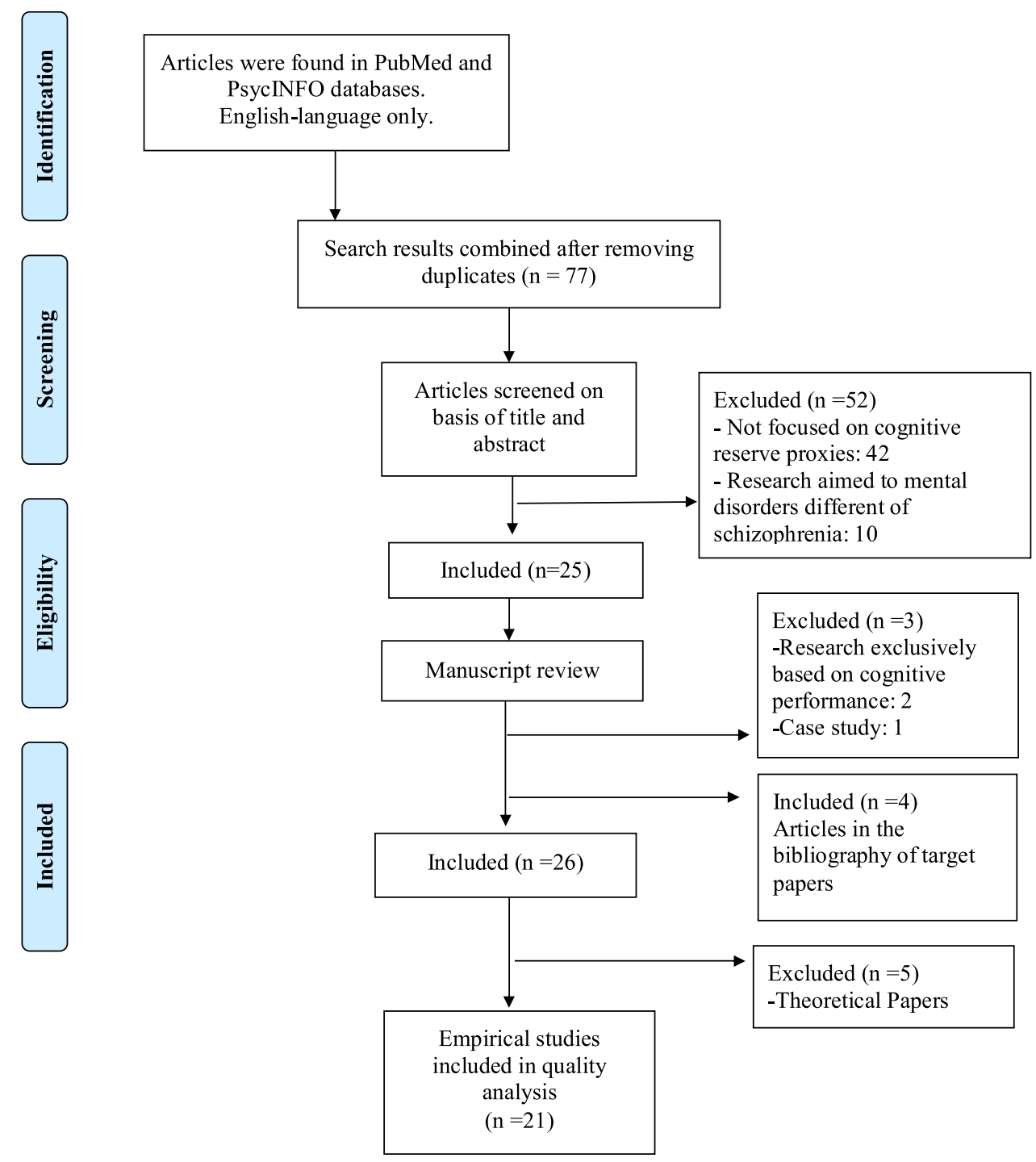

Fig. 1. PRISMA flow chart of the selected information through the phases of the review.

\subsection{CR and risk of schizophrenia}

It has been argued that CR proxies would delay the onset of the symptoms and, therefore, the incidence of schizophrenia. Supporting this idea, several longitudinal cohort studies (Gunnell et al., 2002; Koenen et al., 2009; Zammit et al., 2004) and a 19-year longitudinal community-based study (Kremen et al., 1998) found low intelligence scores in children and adolescents who developed schizophrenia or other psychotic disorders in their adulthood. In the same vein, a metaanalysis of 14 longitudinal studies on the relationship between schizophrenia and premorbid intelligence (Khandaker et al., 2011) confirmed the relationship between IQ at younger age and the later diagnosis of schizophrenia. In this study, it was observed that, for every point of decrease in premorbid IQ compared to the average, the risk of developing schizophrenia increased by $3.7 \%$. Thus, the influence of intelligence seems to be linear over entire IQ range. Furthermore, Khandaker et al. (2011) found an association between lower premorbid IQ and earlier mean age of onset of the disease. Likewise, a poor premorbid adjustment also has been linked to earlier schizophrenia onset (Vourdas et al., 2003), which means that lower social, interpersonal, academic, and occupational functioning before the onset of psychotic symptoms may be an indicator of poor CR (Addington and Addington, 2005; Buonocore et al., 2018b).

Interestingly, Leeson et al. (2012) compared two groups of patients with schizophrenia (cannabis users and never-users) in measures of premorbid function, cognition, and clinical outcomes. $72 \%$ of patients were reassessed 15 months after their initial assessment. The findings reflected that cannabis users had higher current IQ and better cognitive performance (verbal learning, working memory span and planning ability) and social function (Social Function Scale) at the onset of psychosis but no differential group changes in cognition or social function were observed at follow up. Although there was a strong linear relationship between age at first cannabis use and age at onset of both prodromal and psychotic symptoms, a higher IQ may compensate cognitive and social deficits at early stages of psychosis.

\subsection{Influence of $C R$ in the cognitive, functional, and psychopathological} manifestations of schizophrenia

In this section, we review the findings that different studies have obtained regarding each of the factors and life experiences that can influence CR, in order to understand how and on which manifestations of schizophrenia these factors exert their influence.

\subsubsection{Intelligence}

A high level of intelligence after psychosis has been repeatedly associated with less severe cognitive impairment in people with schizophrenia (Holthausen et al., 2002; Kravariti et al., 2003). On the other 
hand, Wang et al. (2016) found that a longer duration of the first episode of psychosis (FEP) was associated with poorer cognitive scores. These authors noted that this association was more pronounced in a subgroup of patients with lower premorbid IQ, but this relation was not found in the high premorbid IQ group. Hence, it seems that a high premorbid intelligence can buffer the adverse effects of psychosis on cognition.

These results allow us to conclude that premorbid IQ influences schizophrenia, but does this disorder, in turn, affect IQ? When the magnitude of the decrease in intelligence was assessed in patients with schizophrenia, using estimates of premorbid IQ, it was found that approximately $40 \%$ suffer a decrease of 10 points or more, while the rest preserve IQ unchanged (Badcock et al., 2005; Joyce et al., 2005; Kremen et al., 2008; Weickert et al., 2000). This heterogeneous pattern in the differences between premorbid IQ and IQ after psychosis was replicated by Leeson et al. (2011) in a three-year follow-up study after the FEP. Their findings indicated that, it is possible to differentiate 3 IQ categories in these patients: preserved average/high IQ, stable low IQ, and deteriorated IQ. According to the CR postulates, patients with a poorer level of intelligence would have lower CR level that those with a superior IQ. Supporting this idea, the low and the deteriorated IQ groups were equally impaired on tests of memory and executive function compared with the preserved IQ and control groups. In addition, patients with deteriorated and low IQ showed more negative and disorganization symptoms and were characterized by a lower score in the Employment/Occupation subscale of the Social Functioning Scale (SFS) than those who retained an average/high IQ. The authors were unable to identify any predictive measures that could distinguish between those who undergo a decline in cognitive ability after FEP and those who preserve a premorbid ability in the average/high range.

Similarly, Holthausen et al. (2002) observed that patients who had preserved normal cognitive function (measured through an extensive neuropsychological battery) had a higher IQ and educational level, in comparison with those who had cognitive deficits. In addition, IQ was also associated with better overall social functioning (better self-care and occupational roles). Thus, level of intelligence may partially explain the cognitive, clinical and functional heterogeneity in people with schizophrenia.

\subsubsection{Education}

Holthausen et al. (2002) also established an association between high education (level of education, from (1) primary school up to (8) university or graduate school) and better cognitive performance in patients with schizophrenia. Consistently, higher education has been associated in several studies with better cognitive-functional performance and more benign clinical progression (less negative symptoms and behavioral disorders) (Amoretti et al., 2016, 2018; de la Serna et al., 2013).

Another current study confirms the association between education and cognition, finding that in patients with schizophrenia who were carriers of the alleles of risk for cognitive dysfunction (ACE D and APOE-4), those who had received more years of education obtained higher scores in verbal memory than those with lower educational level (Ward et al., 2017). These findings suggest that higher CR can mitigate the impact of a genetic polymorphism associated with cognitive dysfunction in schizophrenic patients.

On the other hand, Kanchanatawan et al. (2018b) reported that education could be a protective factor against psychosis. The authors found that schizophrenia patients may be divided into two qualitatively distinct categories "simple neurocognitive psychosis" (SNP), a less welldeveloped phenotype, and "major neurocognitive psychosis" (MNP), the full-blown phenotype or core illness. MNP cluster showed increased negative, psychotic, excitation, hostility, mannerism, depression and anxiety symptoms, and more neuroimmune (changes in IgA/IgM responses to tryptophan catabolites) and cognitive (episodic, spatial working and semantic memory neuropsychological functioning and paired association learning) disorders. These deficiencies were less pronounced in SNP group, which showed significantly higher level of education and more employment in comparison with MNP. These results suggest that education is a protective factor against MNP. This is coherent with recent findings which show that lower education is a vulnerability factor for cognitive impairment and behavioral symptoms in people with psychosis (Kanchanatawan et al., 2018a).

Finally, a study suggested an association between low educational level and soft neurological signs (subtle deficits in motor coordination, sensory integration, complex motor tasks and right/left and spatial orientations) in schizophrenia (Herold et al., 2018). This study showed a greater susceptibility of patients (vs. healthy controls) to the physiological effects of age, which may be buffered by the level cognitive reserve. About this possibility, the authors suggested that more neurological symptoms in chronic patients may be associated with lower educational levels.

\subsubsection{Leisure and social activities}

We found two studies that investigated the impact of leisure and social activities in patients with schizophrenia, including these activities along with premorbid IQ and education in a compound index of CR (Amoretti et al., 2016; de la Serna et al., 2013). In both studies a CR proxy (made up of the three indicators) correctly classified the samples into patients or healthy controls, suggesting that, to some extent, these factors influenced the risk of developing the disease. These studies also indicate the need to include other measures of CR besides premorbid IQ to increase its predictive capacity for cognitive outcomes. More specifically, de la Serna et al. (2013) showed that CR was able to predict better scores on working memory and attention at two-year follow-up. Consistently, Amoretti et al. (2016) also observed that higher CR may help FEP patients to prevent cognitive and functional decline at two years follow-up.

A recent study also included leisure activities as an indicator of CR (Amoretti et al., 2018). The researchers studied the role of CR (premorbid IQ, education-occupation and leisure activities) in the outcome of affective and non- affective psychoses in a FEP sample (2 years follow up). In general, patients with high CR showed a better neurocognitive performance and premorbid functioning. What this paper provides as a novelty is the discovery that the predictive quality of CR in the outcome of psychoses depends on diagnosis (affective or non-affective) and the proposal of individualized rehabilitation treatments based on the type of psychosis. About the role of $\mathrm{CR}$, results showed that in non-affective psychosis, CR predicted functioning (FAST and GAF), clinical (PANSS$\mathrm{N}$; Positive and Negative Syndrome Scale-Negative) and cognitive (verbal memory, attention span, working memory, and global cognition) performance; while in affective psychosis, CR predicted cognitive performance, but not functionality or clinical outcomes. Moreover, in non-affective psychosis, CR only explained a low variance percentage of the result both in functionality and in symptomatology, so the article raises that there are other variables like individual characteristics and antipsychotics dose that need attention.

\subsubsection{Premorbid adjustment}

Some studies have used premorbid adjustment (Premorbid Adjustment Scale, PAS) as CR indicator, finding an association with different cognitive outcomes. In particular, Cuesta et al. (2015) found that good premorbid adjustment in adolescence was strongly associated with better performance on the different cognitive domains analyzed (i.e., laterality, premorbid IQ, processing speed, executive function, working memory, verbal memory, and social cognition) in patients with schizophrenia. In addition, the parents' high status socioeconomic (SES) (Hollingshead-Redlich Index of Social Position) made also a significant contribution to the variance in laterality, premorbid IQ, attention, processing speed and working memory scores. Buonocore et al. (2018b), also found a positive relationship between PAS peer relationships and adaptation to school subtests and the BACS (Brief 
Assessment for Cognition in Schizophrenia) working memory subtest. However, the authors did not find any correlation between premorbid adjustment and psychopathology measures. A possible explanation is that this investigation did not involve a longitudinal evaluation of the clinical course. Interestingly, the same study also highlighted the role of premorbid adjustment to improvement after rehabilitation.

In addition, a report suggests that patients with poor adjustment have more severe positive and negative symptoms in FEP (Rabinowitz et al., 2002). Similarly, Addington and Addington (2005) found an association between poor premorbid adjustment, increased negative symptoms and poorer social functioning (Quality of Life Scale) two years after the onset of schizophrenia.

\section{3. $C R$ and intervention programs in patients with schizophrenia}

Barlati et al. (2018) found in their review that cognitive remediation interventions seem to be more effective in schizophrenia patients with the following features: younger age, shorter duration of illness, few disorganized symptoms, greater pretreatment cognitive reserve and lower doses antipsychotics in their current treatment. This study support that higher $\mathrm{CR}$ is associated with greater response to cognitive rehabilitation.

Amoretti et al. (2018), previously described, posed different rehabilitation programs according to type of psychosis (affective or nonaffective). In affective psychosis, patients with low CR presented a lower functionality and verbal memory at two year follow-up compared to those with high CR. Thereby, the authors suggest that functional remediation therapy may be an optimal choice for these patients, as this intervention type is the one that has proven to be most effective in improving psychosocial functioning and verbal memory (Bonnin et al., $2016 \mathrm{a}, \mathrm{b})$. On the other hand, in non-affective psychosis, patients with low CR show a worse neuropsychological performance, so an intervention based on cognitive rehabilitation can be suggested for them.

Fiszdon et al. (2006) did not use the term "cognitive reserve" as such, but instead examined the role of a similar concept ("intellectual status", based on the cognitive change that occurs after illness onset) in predicting response to cognitive remediation in a sample of patients diagnosed with schizophrenia or schizoaffective disorder. "Intellectual status" is based on the cognitive change that occurs after illness onset and it is calculated by measuring difference between premorbid and current IQ. Based on Weickert et al. (2000), they classified patients with schizophrenia as follows: preserved IQ (intellectual function did not change from premorbid levels), impaired IQ (intellectual function declined after the onset of the disorder) and compromised IQ (consistently low intellectual function). In the study of Fiszdon et al. (2006), the three groups were randomized to receive either Neurocognitive Enhancement Therapy with Work Therapy (NET + WT) or Work Therapy only (WT only) during six months. The findings showed that subjects in NET + WT showed greater improvement in cognition than those in WT only, but response to cognitive remediation differed by intellectual group. Thus, preserved and deteriorated intellectual groups in the NET + WT condition showed significantly greater improvement in the analysis of pre-post neuropsychological test performance. The authors suggest that these patients may have greater $\mathrm{CR}$ than the compromised group, what would allow them to generalize the benefits of NET to similar but untrained tasks. This article supports the idea that CR affects improvement over cognitive rehabilitation, but this influence seems to be more complicated than it was initially thought.

We found two articles about the relationship between CR and cognitive rehabilitation therapy (CRT; Buonocore et al., 2018b; Kontis et al., 2013). CRT is a psychosocial treatment aimed at improving the cognitive function of patients with schizophrenia (Kontis et al., 2013). It has been postulated that CRT can help improve CR and cognition, reducing symptoms in adults with psychiatric illnesses (Vance et al., 2013). Its positive effects on cognitive, clinical, and functional outcomes in these patients have been shown in several randomized clinical trials (d'Amato et al., 2011; Katsumi et al., 2017; Ventura et al., 2017; Wykes et al., 1999), as well as in a meta-analysis that examines 26 trials of this type (McGurk et al., 2007). However, the degree of cognitive improvement observed varied between studies. Could this variation be, at least partially, due to patients' level of CR? Will patients with high CR show more improvement after CRT? These questions should be analyzed with cautious.

Kontis et al. (2013) explored the influence of age and CR on the improvement after CRT. These authors reported that premorbid IQ was associated with better working memory performance at post-treatment, but this only happened in younger participants ( $<40$ years) regardless of therapy that they received (CRT or treatment as usual). On the other hand, in older patients ( $>40$ years) CRT did not improve cognition at any level of CR. Despite this, their findings support the hypothesis that CR could enhance practice effects in younger patients. However, because this was only found for premorbid IQ, it is possible that different proxies of $\mathrm{CR}$ have different relationships with cognitive performance. We should highlight that Fiszdon et al. (2006) did not directly investigate the role of premorbid IQ on cognitive remediation outcomes, instead they use three levels of "intellectual status" as measure of intelligence. In addition, they did not use the same cognitive program that Kontis et al. (2013), so that both studies are not directly comparable and their results should not be considered contradictory.

Buonocore et al. (2018b) investigated seventy-nine patients with schizophrenia who underwent a combined intervention consisting of 36 CRT sessions added to standard rehabilitation therapy (12 one-hour sessions of social skills training programmes for residential, vocational and recreational functioning). All patients were assessed at baseline for psychopathology, premorbid adjustment, intellectual level, cognition and functioning. Cognitive evaluations were also repeated after intervention. The results showed that premorbid adjustment along with age can predict degree of improvement obtained after applying CRT, which contradicts the results obtained by Kontis et al. (2013).

Apart from NET, CRT and WK, there are other treatment approaches for patients with schizophrenia, like socio-cognitive rehabilitation trainings. Thus, Buonocore et al. (2018a) analyzed the effect of CR (current IQ and premorbid functioning) on theory of mind improvement after theory of mind intervention (ToMI) or social cognition and interaction training (SCIT). Sixty patients were treated for three months of ToMI (18 sessions of $1 \mathrm{~h}$, using comic strips and cartoons depicting human social interactions) or SCIT (12 sessions of $1 \mathrm{~h}$, using short videos depicting human social interactions, selected from international movies) and six months of standard rehabilitation therapy. A cluster analysis showed three relevant profiles of CR which are similar to the three groups defined by Leeson et al. (2011): low early premorbid functioning and mild intellectual impairment (cluster $1: \downarrow P A S \pm$ IQ), average/high early premorbid functioning trend with moderate intellectual impairment (cluster 2: \pm PAS $\downarrow$ IQ) and good early premorbid functioning associated to IQ within normal limits (cluster 3: $\uparrow$ PAS $\uparrow$ IQ). In this study, no relationship was found between CR clusters and clinical and neurocognitive baseline measures. The results also showed that there was a significant effect of CR on both baseline ToM performance and treatment outcome: patients with higher CR reached significantly better ToM scores. Specifically, cluster 1 showed a high degree of improvement to the point that post-treatment scores were comparable to those achieved by Group 3 with higher CR and better ToM performance at baseline. Patients belonging to Cluster 2 also obtained a significant improvement, but their performance remained significantly lower than Cluster 3. The latter did not show a significant change after treatment due to possible ceiling effect. This work partially supports our hypothesis, as although that CR may influence also specific rehabilitation outcome, it seems that the relationship is not exactly as we raised it. One possible explanation is that effect of intelligence and premorbid functioning should be assessed independently. 


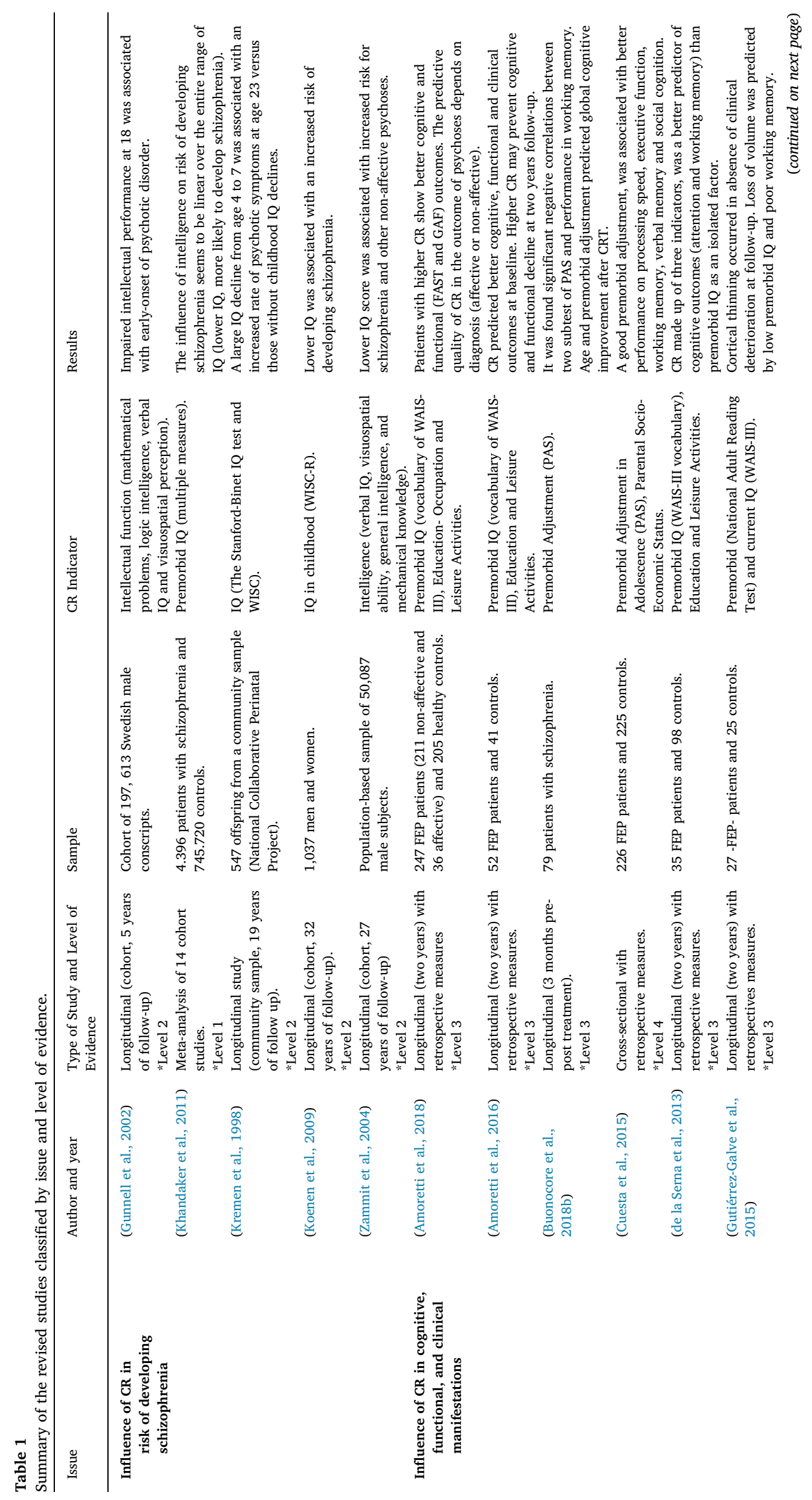




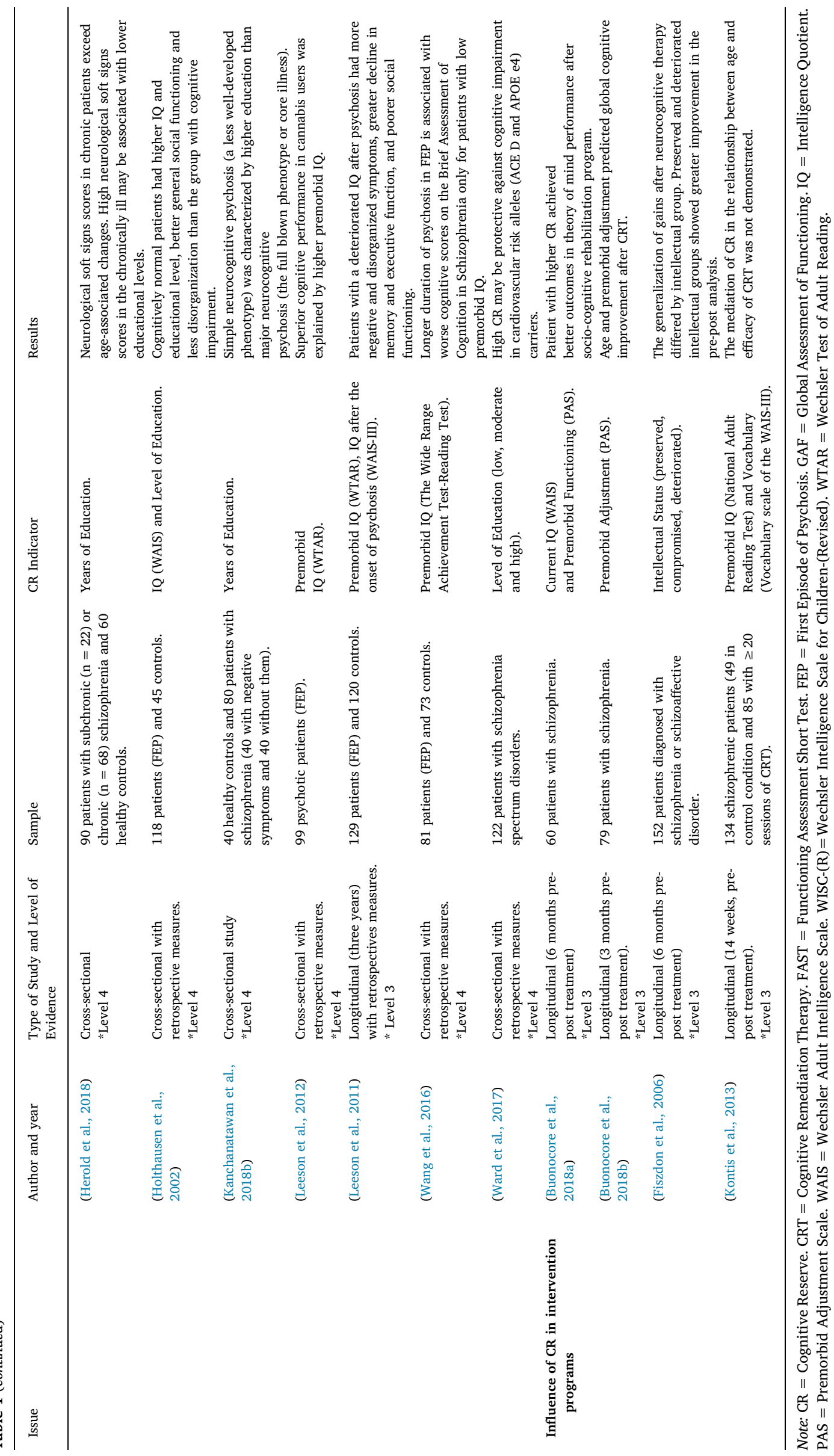




\section{Discussion}

The CR hypothesis has been generally framed in the neurodegenerative paradigm associated with $\mathrm{AD}$, where high $\mathrm{CR}$ can act as a protective mechanism against the development and expression of cognitive and functional symptoms. In addition, higher CR is associated with a faster progression of dementia due to the advanced neuropathological state when the begin cognitive changes. In schizophrenia, high CR can protect the individual against the development of the disorder and buffer its symptoms. However, the symptomatic evolution in schizophrenia does not conform to the slow progression typical of AD (neurodegenerative hypothesis), and significant variability is found in the rate of patients' decline after the diagnosis despite the verification of brain damage. In fact, this rate of decline is related to premorbid factors such as IQ, which is associated with slower deterioration in cognitive and social functioning. Alternatively, the neurodevelopmental hypothesis suggests the presence of a cerebral dysfunction before the onset of the illness, which opens the door to psychosocial interventions that slow down or decrease the cognitive and functional deficits after diagnosis.

The reviewed empirical evidence allows us to support two of the three hypotheses raised above. Different factors associated with CR can modify the process of neurodevelopment in schizophrenia. If CR is also a protective factor against the development of schizophrenia and reduces the impact of the clinical manifestations of the disease. According to the analysis of the evidence (level 1), a low premorbid IQ is related to an increased risk of developing schizophrenia (Khandaker et al., 2011). In this context, evidence about other single CR proxies is very limited, but some studies (level 3) confirm that a greater CR protects against the development of schizophrenia (Amoretti et al., 2016, 2018; de la Serna et al., 2013; Koenen et al., 2009). Moreover, individual CR measures (premorbid adjustment, years of education, socioeconomic status) and compounds (IQ, education, social activities) have been related to a better symptomatic progression of cognitive and behavioral symptoms associated with schizophrenia, but only levels 3 and 4 of evidence were reached in the analyzed studies.

With regard to the third hypothesis raised about the possibility that greater CR increases the benefit of cognitive rehabilitation in patients with schizophrenia, studies are scarce and the results have to be examined carefully. Several evidences at level 3 suggest a relationship between different clusters of intelligence and intervention benefits (Fiszdon et al., 2006; Buonocore et al., 2018a,b). On the other hand, Kontis et al. (2013) observed that CR proxies did not modify CRT treatment effect and that older people with schizophrenia do not seem to benefit from the CRT. Thus, current data do not allow us to confirm our initial hypothesis, although it seems to indicate that CR has an influence on benefits obtained from rehabilitation programs. It should be noted that groups with different CR level were typically defined in post-hoc analysis, so it is necessary to carry out further controlled and randomized clinical trials to test differential effects of interventions.

The present review leaves other questions to be addressed in future research. One report mentioned above (Gutiérrez-Galve et al., 2015) found that there was less thinning of the frontal cortex in patients with higher IQ (premorbid and current) and with better working memory. Thus, it is proposed that patients with lower CR could be more vulnerable to the development of cortical abnormalities when they are exposed to medications or other biological factors associated with the disease. In relation to this hypothesis, the article of Herold et al. (2018) suggest an association between low educational level and frequency of neurological symptoms in chronic schizophrenia. It would be interesting to clarify this relationship in a longitudinal analysis of brain changes.

Otherwise, Kanchanatawan et al. (2018b) showed that education seems to be a protective factor against MNP, opening new lines of research and reflecting the needed of more studies about this issue. Another unanswered question in this review is why premorbid intelligence decreases after the FEP in some patients and not in others, and whether this reduction occurs at a specific point in the IQ continuum or across the entire continuum. It would be interesting to study the possible moderation of genetic, educational, occupational and other factors in the cognitive change during the development of psychosis. Schizophrenia has been linked to a multitude of genes associated with brain interconnectivity. It is possible that the change in the activity of these genes is related to a greater or lesser decline (Akbarian, 2014; Dempster et al., 2013). Studies focusing on these questions could shed light on the prognosis of patients and genetic interaction with factors associated with brain neuroplasticity. Further, this review points out the need for prospective studies and controlled randomized clinical trials to control the possibilities of reverse causation bias between $\mathrm{CR}$ proxies and the outcomes. Another issue that should be studied in greater depth in future investigations is the influence of factors such as socioeconomic status and the differential weighting of each factor in the development and prognosis of schizophrenia should be taken into account in the future.

Finally, the results of this review pose an interesting question: why do older people with schizophrenia not seem to benefit from CRT? Despite the opposing evidence (Eyler Zorrilla et al., 2000), it has been suggested that cognitive functioning in schizophrenic patients suffers a more rapid decline after 50 years of age with respect to healthy subjects and patients with $\mathrm{AD}$, in whom the cognitive impairment occurs regardless of age (Friedman et al., 2001). This rapid decline could make CRT in these patients ineffective. However, they could benefit from other treatments. In this sense, Bell, Fiszdon, Greig and Bryson (2005) showed that, despite greater neurocognitive compromise, older people ( $>50$ years) with schizophrenia included in a 6-month work therapy program had fewer symptoms in the PANSS and improved quality of life (Quality of Life Scale) - at least as much as young patients. It is well known that occupational experiences impart reserve against the expression of Alzheimer's pathology (Stern, 2012; Valenzuela and Sachdev, 2005). In particular, work therapy could even act as a vital experience that improves the CR against the manifestations of schizophrenia, reducing symptoms and improving the quality of life (Bell et al., 2005). However, a causal relationship between occupation and clinical improvements cannot be established due to the absence of a control group. In the future, new studies incorporating control groups without treatment versus groups of patients with different levels of CR receiving CRT, work therapy and other treatments are also necessary to check if premorbid factors can affect the effectiveness of treatment, and if so, discover which of them and how they do so. In particular, we want to highlight the importance of developing more research with old patients, who do not seem to benefit from the CRT. They might need a different approach to cognitive remediation to optimize specific benefits in this population. It is also important to continue studying CR profiles, since they could be used to personalize interventions, as noted by Buonocore et al. (2018a).

This work has a number of limitations that should be considered when evaluating the findings. First, the vast majority of studies employed retrospective measures, limiting the scope of the results obtained. In the same vein, we believe that it is necessary to perform longitudinal studies of the greatest possible duration in order to better understand the prognosis of this disease throughout the life cycle. Second, different methods are currently used to estimate CR, which made comparisons between studies problematic. Thus, some studies calculated complex CR indexes, combining different estimators, whereas others used single proxies. In this context, it has been shown that education, occupation and leisure activities may independently contribute to the development of CR (Foubert-Samier et al., 2012). However, Grotz et al. (2017) demonstrated that indexes, taking into account specific weight of the main CR proxies, may possibly be a more accurate approach to estimate individuals' CR. Inevitably, some CR proxies are correlated (e.g., IQ and educational achievement), which emphasizes the need to assess the single contributions of proxies on 
different outcomes. Third, cross-sectional studies do not allow us to differentiate whether education (or other single proxies) acts through compensation mechanism against the brain damage, or is simply a sociocultural variable associated with better cognitive and functional performance. Although the effect of education on dementia prevention has been consistently demonstrated (Meng and D'Arcy, 2012), longitudinal evidence on schizophrenia is inexistent. Finally, we cannot ignore that the inclusion of other psychotic patients who do not have schizophrenia in some studies, such as bipolar and schizotypal disorder, could influence the results. Amoretti et al. (2018) found differences in the predictive quality of $\mathrm{CR}$ on psychosis outcomes depending on diagnosis (affective or non-affective psychosis). In addition, they proposed two lines of treatment based on the type of psychosis. It is necessary to clarify if this differentiation is justified or not by developing more studies focused on this point.

In conclusion, determining how different $\mathrm{CR}$ proxies influence schizophrenia may be relevant for identifying individuals who may be at risk of suffering from this disorder and for implementing preventive programs during the early course of the illness. Incorporating measures of CR would also allow improved accuracy of patient prognoses and the development of personalized rehabilitation programs that focus on the domains that are expected to be most impaired in the course of the disease. In this regard, the creation of operational and well-validated tools to estimate CR is a challenge in current research.

\section{Declaration of Competing Interest}

The authors have not reported any conflict of interest.

\section{Acknowledgments}

We thank suggestions of Dr. J. López-Lucas for database selection and execution of revision process.

\section{References}

Addington, J., Addington, D., 2005. Patterns of premorbid functioning in first episode psychosis: relationship to 2-year outcome. Acta Psychiatr. Scand. 112 (1), 40-46. https://doi.org/10.1111/j.1600-0447.2005.00511.x.

Akbarian, S., 2014. Epigenetic mechanisms in schizophrenia. Dialogues Clin. Neurosci. 16 (3), 405-417.

Amoretti, S., Bernardo, M., Bonnin, C.M., Bioque, M., Cabrera, B., Mezquida, G., et al., 2016. The impact of cognitive reserve in the outcome of first-episode psychoses: 2 year follow-up study. Eur. Neuropsychopharmacol. 26 (10), 1638-1648. https://doi. org/10.1016/j.euroneuro.2016.07.003.

Amoretti, S., Cabrera, B., Torrent, C., Mezquida, G., Lobo, A., González-Pinto, A., et al., 2018. Cognitive reserve as an outcome predictor: first-episode affective versus nonaffective psychosis. Acta Psychiatr. Scand. 138 (5), 441-455. https://doi.org/10. 1111/acps.12949.

Badcock, J.C., Dragović, M., Waters, F.A.V., Jablensky, A., 2005. Dimensions of intelligence in schizophrenia: evidence from patients with preserved, deteriorated and compromised intellect. J. Psychiatr. Res. 39 (1), 11-19. https://doi.org/10.1016/j. jpsychires.2004.05.002.

Barnett, J.H., Salmond, C.H., Jones, P.B., Sahakian, B.J., 2006. Cognitive reserve in neuropsychiatry. Psychol. Med. 36 (8), 1053. https://doi.org/10.1017/ S0033291706007501.

Barlati, S., Deste, G., Galluzzo, A., Perin, A.P., Valsecchi, P., Turrina, C., Vita, A., 2018 Factors associated with response and resistance to cognitive remediation in schizophrenia: a critical review. Front. Pharmacol. 9, 1542. https://doi.org/10.3389/fphar. 2018.01542.

Bell, M.D., Fiszdon, J.M., Greig, T.C., Bryson, G.J., 2005. Can older people with schizophrenia benefit from work rehabilitation? J. Nerv. Ment. Dis. 193 (5), 293-301. https://doi.org/10.1097/01.nmd.0000161688.47164.71.

Bonnin, C.M., Reinares, M., Martínez-Arán, A., Balanzá-Martínez, V., Sole, B., Torrent, C., et al., 2016a. Effects of functional remediation on neurocognitively impaired bipolar patients: enhancement of verbal memory. Psychol. Med. 46 (2), 291-301. https:// doi.org/10.1017/S0033291715001713.

Bonnin, C.M., Torrent, C., Arango, C., Amann, B.L., Solé, B., González-Pinto, A., et al., 2016b. Functional remediation in bipolar disorder: 1-year follow-up of neurocognitive and functional outcome. Br. J. Psychiatry 208 (1), 87-93. https://doi.org/10. 1192/bjp.bp.114.162123.

Bora, E., 2015. Neurodevelopmental origin of cognitive impairment in schizophrenia. Psychol. Med. 45 (1), 1-9. https://doi.org/10.1017/S0033291714001263.

Bora, E., Murray, R.M., 2014. Meta-analysis of cognitive deficits in ultra-high risk to psychosis and first-episode psychosis: do the cognitive deficits progress over, or after, the onset of psychosis? Schizophr. Bull. 40 (4), 744-755. https://doi.org/10.1093/ schbul/sbt085.

Bryson, G.J., Silverstein, M.L., Nathan, A., Stephen, L., 1993. Differential rate of neuropsychological dysfunction in psychiatric disorders: comparison between the Halstead-Reitan and Luria-Nebraska batteries. Percept. Mot. Skills 76 (1), 305-306.

Buonocore, M., Bechi, M., Uberti, P., Spangaro, M., Cocchi, F., Guglielmino, C., et al., 2018a. Cognitive reserve profiles in chronic schizophrenia: effects on theory of mind performance and improvement after training. J. Int. Neuropsychol. Soc. 24 (6), 563-571. https://doi.org/10.1017/S1355617718000012.

Buonocore, M., Bosinelli, F., Bechi, M., Spangaro, M., Piantanida, M., Cocchi, F., et al., 2018 b. The role of premorbid adjustment in schizophrenia: Focus on cognitive remediation outcome. Neuropsychol. Rehabil. 1-14. https://doi.org/10.1080/ 09602011.2018 .1433048$.

Burke, S.N., Barnes, C.A., 2006. Neural plasticity in the ageing brain. Nat. Rev. Neurosci. 7 (1), 30-40. https://doi.org/10.1038/nrn1809.

Cohen, A.S., Saperstein, A.M., Gold, J.M., Kirkpatrick, B., Carpenter, W.T., Buchanan, R.W., 2007. Neuropsychology of the deficit syndrome: new data and meta-analysis of findings to date. Schizophr. Bull. 33 (5), 1201-1212. https://doi.org/10.1093/ schbul/sbl066.

Concato, J., Shah, N., Horwitz, R.I., 2000. Randomized, controlled trials, observational studies, and the hierarchy of research designs. N. Engl. J. Med. 342 (25), 1887-1892.

Contador, I., Bermejo-Pareja, F., Pablos, D.L., Villarejo, A., Benito-León, J., 2017. High education accelerates cognitive decline in dementia: a brief report from the population-based NEDICES cohort. Dement. Neuropsychol. 11 (3), 297-300. https://doi. org/10.1590/1980-57642016dn11-030012.

Contador, I., Bermejo-Pareja, F., Puertas-Martin, V., Benito-Leon, J., 2015. Childhood and adulthood rural residence increases the risk of dementia: NEDICES study. Curr. Alzheimer Res. 12 (4), 350-357.

Contador, I., Fernández-Calvo, B., Ramos, F., Olazarán, J., 2016. Influence of educational attainment on cognition-based intervention programs for persons with mild Alzheimer's disease. J. Int. Neuropsychol. Soc. 22 (5), 577-582. https://doi.org/10. 1017/S135561771600014X.

Cuesta, M.J., Sánchez-Torres, A.M., Cabrera, B., Bioque, M., Merchán-Naranjo, J., Corripio, I., et al., 2015. Premorbid adjustment and clinical correlates of cognitive impairment in first-episode psychosis. The PEPsCog study. Schizophr. Res. 164 (1-3), 65-73. https://doi.org/10.1016/j.schres.2015.02.022.

d'Amato, T., Bation, R., Cochet, A., Jalenques, I., Galland, F., Giraud-Baro, E., et al., 2011. A randomized, controlled trial of computer-assisted cognitive remediation for schizophrenia. Schizophr. Res. 125 (2-3), 284-290. https://doi.org/10.1016/j.schres. 2010.10.023.

Davidson, M., Reichenberg, A., Rabinowitz, J., Weiser, M., Kaplan, Z., Mark, M., 1999. Behavioral and intellectual markers for schizophrenia in apparently healthy male adolescents. Am. J. Psychiatry 156 (9), 1328-1335. https://doi.org/10.1176/ajp. 156.9.1328.

de la Serna, E., Andrés-Perpiñá, S., Puig, O., Baeza, I., Bombin, I., Bartrés-Faz, D., et al., 2013. Cognitive reserve as a predictor of two-year neuropsychological performance in early onset first-episode schizophrenia. Schizophr. Res. 143 (1), 125-131. https:// doi.org/10.1016/j.schres.2012.10.026.

DeLisi, L.E., 1992. The significance of age of onset for schizophrenia. Schizophr. Bull. 18 (2), 209-215. https://doi.org/10.1093/schbul/18.2.209.

DeLisi, L.E., Sakuma, M., Ge, S., Kushner, M., 1998. Association of brain structural change with the heterogeneous course of schizophrenia from early childhood through five years subsequent to a first hospitalization. Psychiatry Res. 84 (2-3), 75-88.

Dempster, E., Viana, J., Pidsley, R., Mill, J., 2013. Epigenetic studies of schizophrenia: progress, predicaments, and promises for the future. Schizophr. Bull. 39 (1), 11-16. https://doi.org/10.1093/schbul/sbs139.

Eyler Zorrilla, L.T., Heaton, R.K., McAdams, L.A., Zisook, S., Harris, M.J., Jeste, D.V., 2000. Cross-sectional study of older outpatients with schizophrenia and healthy comparison subjects: no differences in age-related cognitive decline. Am. J. Psychiatry 157 (8), 1324-1326. https://doi.org/10.1176/appi.ajp.157.8.1324.

Foubert-Samier, A., Catheline, G., Amieva, H., Dilharreguy, B., Helmer, C., Allard, M., Dartigues, J.-F., 2012. Education, occupation, leisure activities, and brain reserve: a population-based study. Neurobiol. Aging 33 (2), 423. https://doi.org/10.1016/j. neurobiolaging.2010.09.023. e15-423.e25.

Friedman, J.I., Harvey, P.D., Coleman, T., Moriarty, P.J., Bowie, C., Parrella, M., et al., 2001. Six-year follow-up study of cognitive and functional status across the lifespan in schizophrenia: a comparison with Alzheimer's disease and normal aging. Am. J. Psychiatry 158 (9), 1441-1448. https://doi.org/10.1176/appi.ajp.158.9.1441.

Fiszdon, J.M., Choi, J., Bryson, G.J., Bell, M.D., 2006. Impact of intellectual status on response to cognitive task training in patients with schizophrenia. Schizophr. Res. 87 (1-3), 261-269. https://doi.org/10.1016/j.schres.2006.04.011.

Green, M.F., Satz, P., Gaier, D.J., Ganzell, S., Kharabi, F., 1989. Minor physical anomalies in schizophrenia. Schizophr. Bull. 15 (1), 91-99.

Grotz, C., Seron, X., Van Wissen, M., Adam, S., 2017. How should proxies of cognitive reserve be evaluated in a population of healthy older adults? Int. Psychogeriatr. 29 (1), 123-136. https://doi.org/10.1017/S1041610216001745.

Gunnell, D., Harrison, G., Rasmussen, F., Fouskakis, D., Tynelius, P., 2002. Associations between premorbid intellectual performance, early-life exposures and early-onset schizophrenia. Cohort study. Br. J. Psychiatry: J. Mental Sci. 181, 298-305.

Gur, R.E., Cowell, P., Turetsky, B.I., Gallacher, F., Cannon, T., Bilker, W., Gur, R.C., 1998 A follow-up magnetic resonance imaging study of schizophrenia. Relationship of neuroanatomical changes to clinical and neurobehavioral measures. Arch. Gen. Psychiatry 55 (2), 145-152.

Gutiérrez-Galve, L., Chu, E.M., Leeson, V.C., Price, G., Barnes, T.R.E., Joyce, E.M., Ron, M.A., 2015. A longitudinal study of cortical changes and their cognitive correlates in patients followed up after first-episode psychosis. Psychol. Med. 45 (1), 205-216. 
https://doi.org/10.1017/S0033291714001433.

Harvey, P.D., Koren, D., Reichenberg, A., Bowie, C.R., 2006. Negative symptoms and cognitive deficits: what is the nature of their relationship? Schizophr. Bull. 32 (2), 250-258. https://doi.org/10.1093/schbul/sbj011.

Herold, C.J., Lässer, M.M., Seidl, U.W., Hirjak, D., Thomann, P.A., Schröder, J., 2018. Neurological soft signs and psychopathology in chronic schizophrenia: a cross-sectional study in three age groups. Front. Psychiatry 9, 98. https://doi.org/10.3389/ fpsyt.2018.00098.

Ho, B.C., Andreasen, N.C., Nopoulos, P., Arndt, S., Magnotta, V., Flaum, M., 2003. Progressive structural brain abnormalities and their relationship to clinical outcome: a longitudinal magnetic resonance imaging study early in schizophrenia. Arch. Gen. Psychiatry 60 (6), 585. https://doi.org/10.1001/archpsyc.60.6.585.

Hoff, A.L., Svetina, C., Shields, G., Stewart, J., DeLisi, L.E., 2005. Ten year longitudinal study of neuropsychological functioning subsequent to a first episode of schizophrenia. Schizophr. Res. 78 (1), 27-34. https://doi.org/10.1016/j.schres.2005.05. 010.

Holthausen, E.A.E., Wiersma, D., Sitskoorn, M.M., Hijman, R., Dingemans, P.M., Schene, A.H., van den Bosch, R.J., 2002. Schizophrenic patients without neuropsychological deficits: subgroup, disease severity or cognitive compensation? Psychiatry Res. 112 (1), 1-11.

Jacobsen, L.K., Giedd, J.N., Castellanos, F.X., Vaituzis, A.C., Hamburger, S.D., Kumra, S., et al., 1998. Progressive reduction of temporal lobe structures in childhood-onset schizophrenia. Am. J. Psychiatry 155 (5), 678-685. https://doi.org/10.1176/ajp. 155.5.678.

Jiménez, M.P., 2016. Plasticidad, compensación y reserva cognitiva y cerebral en el envejecimiento. In: Ballesteros, S. (Ed.), Factores protectores del envejecimiento cog nitivo. Universidad Nacional de Educación a Distancia, Madrid, Spain, pp. 55-86.

Jones, P.B., Rantakallio, P., Hartikainen, A.L., Isohanni, M., Sipila, P., 1998. Schizophrenia as a long-term outcome of pregnancy, delivery, and perinatal complications: a 28-year follow-up of the 1966 north Finland general population birth cohort. Am. J. Psychiatry 155 (3), 355-364. https://doi.org/10.1176/ajp.155.3.355.

Jones, P., Rodgers, B., Murray, R., Marmot, M., 1994. Child development risk factors for adult schizophrenia in the British 1946 birth cohort. Lancet (London, England) 344 (8934), 1398-1402.

Joyce, E.M., Hutton, S.B., Mutsatsa, S.H., Barnes, T.R.E., 2005. Cognitive heterogeneity in first-episode schizophrenia. Br. J. Psychiatry 187 (6), 516-522. https://doi.org/10. 1192/bjp.187.6.516.

Kanchanatawan, B., Sriswasdi, S., Thika, S., Sirivichayakul, S., Carvalho, A.F., Geffard, M., et al., 2018a. Deficit schizophrenia is a discrete diagnostic category defined by neuro-immune and neurocognitive features: results of supervised machine learning. Metab. Brain Dis. 33 (4), 1053-1067. https://doi.org/10.1007/s11011-018-0208-4.

Kanchanatawan, B., Sriswasdi, S., Thika, S., Stoyanov, D., Sirivichayakul, S., Carvalho, A.F., et al., 2018b. Towards a new classification of stable phase schizophrenia into major and simple neuro-cognitive psychosis: results of unsupervised machine learning analysis. J. Eval. Clin. Pract. 24 (4), 879-891. https://doi.org/10.1111/jep. 12945.

Katsumi, A., Hoshino, H., Fujimoto, S., Yabe, H., Ikebuchi, E., Nakagome, K., Niwa, S.-I., 2017. Effects of cognitive remediation on cognitive and social functions in individuals with schizophrenia. Neuropsychol. Rehabil. 1-13. https://doi.org/10. 1080/09602011.2017.1409639.

Katzman, R., 1993. Education and the prevalence of dementia and Alzheimer's disease. Neurology 43 (1, Part 1). https://doi.org/10.1212/WNL.43.1_Part_1.13. 13-13.

Kenny, J.T., Friedman, L., Findling, R.L., Swales, T.P., Strauss, M.E., Jesberger, J.A., Schulz, S.C., 1997. Cognitive impairment in adolescents with schizophrenia. Am. J. Psychiatry 154 (11), 1613-1615. https://doi.org/10.1176/ajp.154.11.1613.

Khandaker, G.M., Barnett, J.H., White, I.R., Jones, P.B., 2011. A quantitative meta-analysis of population-based studies of premorbid intelligence and schizophrenia. Schizophr. Res. 132 (2-3), 220-227. https://doi.org/10.1016/j.schres.2011.06.017.

Koenen, K.C., Moffitt, T.E., Roberts, A.L., Martin, L.T., Kubzansky, L., Harrington, H., et al., 2009. Childhood IQ and adult mental disorders: a test of the cognitive reserve hypothesis. Am. J. Psychiatry 166 (1), 50-57. https://doi.org/10.1176/appi.ajp. 2008.08030343.

Konstantakopoulos, G., Ploumpidis, D., Oulis, P., Patrikelis, P., Soumani, A., Papadimitriou, G.N., Politis, A.M., 2011. Apathy, cognitive deficits and functional impairment in schizophrenia. Schizophr. Res. 133 (1-3), 193-198. https://doi.org/ 10.1016/j.schres.2011.07.003.

Kontis, D., Huddy, V., Reeder, C., Landau, S., Wykes, T., 2013. Effects of age and cognitive reserve on cognitive remediation therapy outcome in patients with schizophrenia. Am. J. Geriatr. Psychiatry 21 (3), 218-230. https://doi.org/10.1016/j.jagp.2012.12. 013.

Kravariti, E., Morris, R.G., Rabe-Hesketh, S., Murray, R.M., Frangou, S., 2003. The Maudsley early onset schizophrenia study: cognitive function in adolescents with recent onset schizophrenia. Schizophr. Res. 61 (2-3), 137-148.

Kremen, W.S., Seidman, L.J., Faraone, S.V., Toomey, R., Tsuang, M.T., 2000. The paradox of normal neuropsychological function in schizophrenia. J. Abnorm. Psychol. 109 (4), 743-752.

Kremen, W.S., Buka, S.L., Seidman, L.J., Goldstein, J.M., Koren, D., Tsuang, M.T., 1998. IQ decline during childhood and adult psychotic symptoms in a community sample: a 19-year longitudinal study. Am. J. Psychiatry 155 (5), 672-677. https://doi.org/10. 1176/ajp.155.5.672.

Kremen, W.S., Seidman, L.J., Faraone, S.V., Tsuang, M.T., 2008. IQ decline in crosssectional studies of schizophrenia: methodology and interpretation. Psychiatry Res. 158 (2), 181-194. https://doi.org/10.1016/j.psychres.2006.01.022.

Kurtz, M.M., 2005. Neurocognitive impairment across the lifespan in schizophrenia: an update. Schizophr. Res. 74 (1), 15-26. https://doi.org/10.1016/j.schres.2004.07. 005 .
Leeson, V.C., Harrison, I., Ron, M.A., Barnes, T.R.E., Joyce, E.M., 2012. The effect of cannabis use and cognitive reserve on age at onset and psychosis outcomes in firstepisode schizophrenia. Schizophr. Bull. 38 (4), 873-880. https://doi.org/10.1093/ schbul/sbq153.

Leeson, V.C., Sharma, P., Harrison, M., Ron, M.A., Barnes, T.R.E., Joyce, E.M., 2011. IQ trajectory, cognitive reserve, and clinical outcome following a first episode of psychosis: a 3-year longitudinal study. Schizophr. Bull. 37 (4), 768-777. https://doi.org/ 10.1093/schbul/sbp143.

Lewis, D.A., Lieberman, J.A., 2000. Catching up on schizophrenia: natural history and neurobiology. Neuron 28 (2), 325-334.

Lieberman, J.A., Sheitman, B.B., Kinon, B.J., 1997. Neurochemical sensitization in the pathophysiology of schizophrenia: deficits and dysfunction in neuronal regulation and plasticity. Neuropsychopharmacology 17 (4), 205-229. https://doi.org/10. 1016/S0893-133X(97)00045-6.

Llamas-Velasco, S., Contador, I., Villarejo-Galende, A., Lora-Pablos, D., Bermejo-Pareja, F., 2015. Physical activity as protective factor against dementia: a prospective population-based study (NEDICES). J. Int. Neuropsychol. Soc. 21 (10), 861-867. https://doi.org/10.1017/S1355617715000831.

Mané, A., Falcon, C., Mateos, J.J., Fernandez-Egea, E., Horga, G., Lomeña, F., et al., 2009. Progressive gray matter changes in first episode schizophrenia: a 4-year longitudinal magnetic resonance study using VBM. Schizophr. Res. 114 (1-3), 136-143. https:// doi.org/10.1016/j.schres.2009.07.014.

McGurk, S.R., Twamley, E.W., Sitzer, D.I., McHugo, G.J., Mueser, K.T., 2007. A metaanalysis of cognitive remediation in schizophrenia. Am. J. Psychiatry 164 (12), 1791-1802. https://doi.org/10.1176/appi.ajp.2007.07060906.

Meng, X., D'Arcy, C., 2012. Education and dementia in the context of the cognitive reserve hypothesis: a systematic review with meta-analyses and qualitative analyses. PLoS One 7, e38268.

Moher, D., Liberati, A., Tetzlaff, J., Altman, D.G., The PRISMA Group, 2009. Preferred reporting items for systematic reviews and meta-analyses: the PRISMA statement. PLoS Med. 6 (7), e1000097. https://doi.org/10.1371/journal.pmed1000097.

OCEBM Levels of Evidence Working Group, 2011. The Oxford 2011 Levels of Evidence. Oxford Centre for Evidence-Based Medicine.

Pantelis, C., Velakoulis, D., McGorry, P.D., Wood, S.J., Suckling, J., Phillips, L.J., et al., 2003. Neuroanatomical abnormalities before and after onset of psychosis: a crosssectional and longitudinal MRI comparison. Lancet 361 (9354), 281-288. https://doi. org/10.1016/S0140-6736(03)12323-9.

Rabinowitz, J., De Smedt, G., Harvey, P.D., Davidson, M., 2002. Relationship between premorbid functioning and symptom severity as assessed at first episode of psychosis. Am. J. Psychiatry 159 (12), 2021-2026. https://doi.org/10.1176/appi.ajp.159.12. 2021.

Rapoport, J.L., Giedd, J., Kumra, S., Jacobsen, L., Smith, A., Lee, P., et al., 1997. Childhood-onset schizophrenia. Progressive ventricular change during adolescence. Arch. Gen. Psychiatry 54 (10), 897-903.

Rapport, L.J., Brines, D.B., Theisen, M.E., Axelrod, B.N., 1997. Full scale IQ as mediator of practice effects: the rich get richer. Clin. Neuropsychol. 11 (4), 375-380. https://doi. org/10.1080/13854049708400466.

Rund, B.R., 2009. Is there a degenerative process going on in the brain of people with schizophrenia? Front. Hum. Neurosci. 3. https://doi.org/10.3389/neuro.09.036. 2009.

Rund, B.R., 1998. A review of longitudinal studies of cognitive functions in schizophrenia patients. Schizophr. Bull. 24 (3), 425-435.

Scarmeas, N., Levy, G., Tang, M.X., Manly, J., Stern, Y., 2001. Influence of leisure activity on the incidence of Alzheimer's disease. Neurology 57 (12), 2236-2242.

Shea, B.J., Reeves, B.C., Wells, G., Thuku, M., Hamel, C., Moran, J., et al., 2017. AMSTAR 2: a critical appraisal tool for systematic reviews that include randomised or nonrandomised studies of healthcare interventions, or both. Br. Med. J. 358, j4008. https://doi.org/10.1136/bmj.j4008.

Sørensen, H.J., Mortensen, E.L., Reinisch, J.M., Mednick, S.A., 2003. Do hypertension and diuretic treatment in pregnancy increase the risk of schizophrenia in offspring? Am. J. Psychiatry 160 (3), 464-468. https://doi.org/10.1176/appi.ajp.160.3.464.

Sporn, A.L., Greenstein, D.K., Gogtay, N., Jeffries, N.O., Lenane, M., Gochman, P., et al., 2003. Progressive brain volume loss during adolescence in childhood-onset schizophrenia. Am. J. Psychiatry 160 (12), 2181-2189. https://doi.org/10.1176/appi.ajp. 160.12.2181.

Stern, Y., 2012. Cognitive reserve in ageing and Alzheimer's disease. Lancet Neurol. 11 (11), 1006-1012. https://doi.org/10.1016/S1474-4422(12)70191-6.

Stern, Y., Albert, S., Tang, M.X., Tsai, W.Y., 1999. Rate of memory decline in AD is related to education and occupation: cognitive reserve? Neurology 53 (9), 1942-1947.

Stern, Y., 2002. What is cognitive reserve? Theory and research application of the reserve concept. J. Int. Neuropsychol. Soc. 8 (3), 448-460.

Stern, Y., Arenaza-Urquijo, E.M., Bartrés-Faz, D., Belleville, S., Cantilon, M., Chetelat, G., et al., 2018. Whitepaper: defining and investigating cognitive reserve, brain reserve, and brain maintenance. Alzheimer's Dementia. https://doi.org/10.1016/j.jalz.2018. 07.219. (in press).

Valenzuela, M.J., Sachdev, P., 2005. Brain reserve and dementia: a systematic review. Psychol. Med. 36 (4), 441. https://doi.org/10.1017/S0033291705006264.

Vance, D.E., Dodson, J.E., Watkins, J., Kennedy, B.H., Keltner, N.L., 2013. Neurological and psychiatric diseases and their unique cognitive profiles: implications for nursing practice and research. J. Neurosci. Nurs. 45 (2), 77-87. https://doi.org/10.1097/ JNN.0b013e3182829038.

Ventura, J., Subotnik, K.L., Gretchen-Doorly, D., Casaus, L., Boucher, M., Medalia, A., et al., 2017. Cognitive remediation can improve negative symptoms and social functioning in first-episode schizophrenia: a randomized controlled trial. Schizophr. Res. 203, 24-31. https://doi.org/10.1016/j.schres.2017.10.005.

Vita, A., De Peri, L., Silenzi, C., Dieci, M., 2006. Brain morphology in first-episode 
schizophrenia: a meta-analysis of quantitative magnetic resonance imaging studies. Schizophr. Res. 82 (1), 75-88. https://doi.org/10.1016/j.schres.2005.11.004.

Vourdas, A., Pipe, R., Corrigall, R., Frangou, S., 2003. Increased developmental deviance and premorbid dysfunction in early onset schizophrenia. Schizophr. Res. 62 (1-2), $13-22$

Wang, M.Y., Ho, N.F., Sum, M.Y., Collinson, S.L., Sim, K., 2016. Impact of duration of untreated psychosis and premorbid intelligence on cognitive functioning in patients with first-episode schizophrenia. Schizophr. Res. 175 (1-3), 97-102. https://doi.org/ 10.1016/j.schres.2016.04.002.

Ward, K.M., Kraal, A.Z., Flowers, S.A., Ellingrod, V.L., 2017. Cardiovascular pharmacogenomics and cognitive function in patients with schizophrenia. Pharmacotherapy 37
(9), 1122-1130. https://doi.org/10.1002/phar.1968.

Weickert, T.W., Goldberg, T.E., Gold, J.M., Bigelow, L.B., Egan, M.F., Weinberger, D.R., 2000. Cognitive impairments in patients with schizophrenia displaying preserved and compromised intellect. Arch. Gen. Psychiatry 57 (9), 907-913.

Wykes, T., Reeder, C., Corner, J., Williams, C., Everitt, B., 1999. The effects of neurocognitive remediation on executive processing in patients with schizophrenia. Schizophr. Bull. 25 (2), 291-307.

Zammit, S., Allebeck, P., David, A.S., Dalman, C., Hemmingsson, T., Lundberg, I., Lewis, G., 2004. A longitudinal study of premorbid IQ score and risk of developing schizophrenia, bipolar disorder, severe depression, and other nonaffective psychoses. Arch. Gen. Psychiatry 61 (4), 354. https://doi.org/10.1001/archpsyc.61.4.354. 